\title{
Urgences
}

\section{De l'humour et de la poésie}

\section{Gilbert Dupuis}

Numéro 12, 3e trimestre 1984

Spécial humour

URI : https://id.erudit.org/iderudit/025181ar

Aller au sommaire du numéro

Éditeur(s)

Urgences

ISSN

0226-9554 (imprimé)

1927-3924 (numérique)

Découvrir la revue

Citer cet article

Dupuis, G. (1984). De l'humour et de la poésie. Urgences, (12), 7-18.

Ce document est protégé par la loi sur le droit d'auteur. L'utilisation des services d'Érudit (y compris la reproduction) est assujettie à sa politique d'utilisation que vous pouvez consulter en ligne.

https://apropos.erudit.org/fr/usagers/politique-dutilisation/
Cet article est diffusé et préservé par Érudit.

Érudit est un consortium interuniversitaire sans but lucratif composé de l'Université de Montréal, l'Université Laval et l'Université du Québec à Montréal. Il a pour mission la promotion et la valorisation de la recherche. https://www.erudit.org/fr/ 


\section{CHRONIQUE}

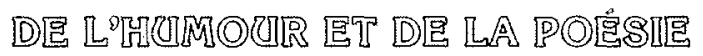

GILBERT DUPUIS 
"Souvenez-vous, avant d'écrire, de la beauté du papier blanc" (Louis Veuillot) (3)

Soit le langage comme milieu de production de l'euphorie littéraire. La parole écrite et le mot parlé. Le verbe porte l'imagination jusqu'à l'oreille. Mais avant, l'oeil du cerveau aura choisi l'angle de vision, le point de vue, la façon de regarder. À la fois spectateur et acteur, l'homme se raconte ses reportages sur lui-même et sur le monde.

\section{la géométrie des regards}

"Les Romains construisèrent de belles routes bien droites pour que leurs ennemis ne puissent pas les attendre au tournant, (2)

On dit que les enfants ont leur langage à eux. Ils ont surtout leur manière de voir. Le monde existe déjà lorsque l'enfant paraît. Le nouvel arrivant exprime spontanément ce qu'il découvre. Son regard n'est pas normalisé, brouillé. On ne lui a pas encore dit quoi voir quand il regarde. II n'est pas encore programmé par la culture. L'enfant de la ville, roulant pour la première fois sur une route de gravier à la campagne, pourra s'exclamer: "Regarde maman, ici, ils ont enlevé l'asphalte! " Ou encore, au moment de la communion, une petite fille: "Dis, maman, tous ces gens à qui on donne un cachet d'aspirine, la musique leur a fait mal à la tête?" (9) Seul l'adulte parle de mots d'enfants.

La Société existe parce qu'un système d'évidences est commun à ses membres. Ces évidences cimentent la cohésion du corps social, lequel en fait une doctrine, une orthodoxie. La Société indique la direction des regards qu'elle privilégie; elle vit du consensus et de l'inflation des axiomes. Et comme on le sait, "un axiome est une chose tellement évidente que ce n'est même pas la peine d'en parler". (2) 
II résulte de tout angle de vision différent de l'angle appris, une modification de l'éclairage qui entraîne une perception différente de la réalité c'est-à-dire un approfondissement de la réalité. Comme l'enfant, "l'humoriste suspend une ou plusieurs évidences" (1) pour en révéler d'autres (au sens photographique) qui, pour avoir été moins éclairées, pourraient s'avérer d'autant plus éclairantes... L'étranger à une Société comme l'enfant au monde adulte sont des naifs du fait qu'ils ignorent les règles du jeu social. Ils ont l'âge psychologique de l'enfant questionneur et s'étonnent des pilules, qu'on veut leur faire avaler avec l'acte de foi en guise de verre d'eau.

Le changement d'éclairage de la réalité n'est pas que conséquence de la "virginité sociale", mais il serait aussi le fait d'un désir relativement conscient. Ce désir force la lecture entre les lignes de la vie, l'examen de l'envers des choses, la simulation des yeux neufs, la recherche d'un nouveau langage pour se dire en "d'autres mots". II s'agit de cette distanciation du contenu vivant par rapport aux contenants que sont la Société, ses règles et ses choses. Il faut voir et faire voir (exprimer) d'une façon efficace pour soi et les autres. II faut aussi dire avec d'autres mots pour voir avec d'autres yeux et vice versa. Car, pour l'enfant d'aujourd'hui, l'oeil n'a pas précédé la caméra...

\section{le langage tricote le monde}

«Martine (quatre ans et demi) voit soudain un magnifique arc-en-ciel: - Regarde, maman, le ciel aussi a froid, mais il a une plus belle écharpe que moin (9)

Les mots savent dessiner, composer des images, s'adresser à l'oeil. Le peintre parlant souhaite approfondir ce qu'il manifeste et ce avec quoi il le fait. Le parleur conscient veut s'approprier un langage de lumière, il désire tutoyer sa langue. 
Pour André Breton et Paul Eluard, la poésie peut se définir comme "l'essai de représenter, ou de restituer, par des cris, des larmes, des caresses..., ou par des objets, ces choses, ou cette chose que tend obscurément d'exprimer le langage articulé dans ce qu'il a d'apparence de vie ou de dessein supposé." (6)

Le langage est l'outil principal de description et de transmission des évidences d'un groupe social. On écrit le code et on codifie par l'écriture. Nul n'ignore plus que le récit d'une réalité n'est pas cette réalité mais la création d'une nouvelle réalité. Nommer les réalités émergentes c'est déjà beaucoup les infléchir. Jusqu'à un certain point, le monde repose sur le langage... de ceux qui le fondent et le diffusent.

La conscience file le nerf conducteur de la vie. L'Homme scrute le tissu de son réel, pour vérifier son envergure et sa vulnérabilité. II se de-tricote pour savoir la force de ses mailles, la chaleur de sa laine et sa sensibilité à l'air du temps. Il a besoin de voyager à rebours de son être pour retrouver sa nudité originelle et son noyau dur. II doit remonter le cours du langage l'ayant constitué pour faire entrer en résonance son sentiment, sa voix et sa soif. L'Homme doit s'intégrer à lui-même.

"La poésie est un moyen de pénétration et d'exploration, une manifestation non pas des réalités objectives qui ne relèvent que de la science mais d'essences concrètes, qui étaient déjà obscurément en nous. Connaissance régressive... dont le critère... est la joie de reconnaittre ce qu'obscurément on pressentait; voilà la vérité de la poésie» (8)

II arrive que le réel soit intolérable pour l'esprit, d'où le besoin de soulager sa conscience (au moins provisoirement) en habitant une autre réalité (une fiction) plus agréable. Cette fiction libérante peut être l'envers du réel douloureux. II y a révolte de l'esprit et métamorphose de la rebellion: à la rescousse voici l'humour, ce "masque du désespoir» (6) humain.

"L'humour est un sentiment complexe où un fond 
comique, produit par la présentation volontairement transposée, et en même temps lucide, de nos idées et de nos sentiments, est très souvent modifié et parfois effacé par une résonance émotionnel/e, morale et philosophique, de nuance très variable, produite par une suggestion générale à laquelle contribuent, les faits présentés, et les milles signes auxquels se révèle l'attitude intérieure de l'humoriste" (M. Cazamian) (1)

L'utilisation particulière du langage, l'entrain de la phrase, le jeu verbal manifestent la poésie ou l'humour; on suscite une euphorie compensatrice en créant une atmosphère de fantaisie, un état second. Le poète/l'humoriste "retournent la réalité sur la doublure" (dédoublement) et s'émeuvent/sourient de ce qu'ils voient/ressentent en se mettant ainsi à la place d'un autre... Ils s'identifient à un jumeau imaginaire!

\section{de la phrase comme d'un train électrique}

"Dis papa, comment ils faisaient les gens pour ne pas dégringoler dans le vide avant qu'on ait voté la loi de la gravitation" (9)

Il y a le jeu avec les mots et le jeu des images. L'exercice de l'humour trouble nos habitudes par les rapprochements inattendus, le dépaysement, la surprise. L'emploi ludique du langage. II s'agit de participer à la transformation d'une réalité en une autre plus accordée à son être profond... du moment. La parole s'amuse à se faire plaisir en jouant sur les tableaux du rythme et de l'inusité.

Nous allons au théâtre de la quotidienneté: nous reconnaissons le décalage entre la parole et le ton, la mise en porte-à-faux du personnage avec la Société, le relief de telle anomalie sur fond de normalité. Le jeu de la tension et de la détente. L'ascension (euphorique) et la chûte (désespérée). On se désintéresse de la réalité extérieure: I'indifférence réduit le sérieux de la vie visible et le nouveau spectateur se dissocie (se joue) du monde. Le Moi se défie du réel qui va de soi... 
"Son humour (celui de Boris Vian) est I'un des domaines où le mélange science-poésie est des plus efficaces, et qui fonctionne suivant les deux schémas inverses. Dans un premier cas, une idée "poétique" singulière, étrange, fulgurante apparaît tout d'abord. Sa description minutieuse, avec ses conséquences diverses et réfléchies, utilise alors toutes les ressources de l'esprit d'analyse de l'ingénieur (...). Dans le second cas, l'observation clinique précède et déclenche l'étincelle poétique: c'est ce qui se produit pour toutes les images émerveillées surgies de l'univers quotidien, et notamment du langage» (4)

Toute écriture est un élan, un désir de sortir de soi, un lieu de distraction fondamentale. Distraire au sens de déranger, de détourner, de "tirer en sens divers». La manière poétique produit le cinéma (images, sons et rythmes) de la connaissance intuitive. Tous les montages sont là pour déjouer le texte prévisible: la surcharge baroque, l'invention surréaliste, la graphie déformée, la répétition amplificatrice, le calembour, l'atténuation, l'ellipse, etc. Ces scènes du feu qui brûle les artifices. Nous songeons ici à cette poésie qui ne recule devant rien pour animer la soirée de sortie. Car le poète est un joueur de tours... de phrase pour son plaisir, pour appartenir à son enfance et n'être pas le jouet de l'existence.

"Le jeu est, de toute évidence, un des besoins fondamentaux de l'enfant et sa satisfaction paroxystique se traduit généralement en éclats de rire (...) S'y justifie p/einement, dès I'origine, la magnifique définition du jeu donnée par Stendhal: une fontaine de surprises" (4)

Outre le mot, l'imagination habite le champ du jeu. Dans le domaine du langage (véhicule privilégié de l'humour et de la poésie), on parle d'imagination pour qualifier l'originalité des moyens employés pour produire sur le lecteur ou l'auditeur l'effet que l'auteur recherche. Pour Henri Baudin, "la fantaisie est à l'imagination ce que la verve est au langage" (4). Sans le frein de l'esprit critique, la fantaisie traverse le rêve et le merveilleux: à force de juxtapositions incohérentes, de surprises, de théories désinvoltes, d'exagérations, de paradoxes, elle 
crée une atmosphère d'irréalité euphorisante. Mais l'enthousiasme ne doit pas faire oublier qu'on ne crée pas à partir de rien et que, pour emprunter à Northrop Frye (10), I'imagination peut être "cette faculté que nous avons d'élaborer des modèles inconsciemment désirés (il dit plausibles) d'expérience humaine».

\section{on dit fou rire et folie douce}

"Le grammairien Bouhours, au moment de mourir, dit aux assistants: Je m'en vais ou je m'en vas. L'un ou l'autre se dit, ou: l'un et l'autre se disent" (3)

L'univers des aliénés aurait pour eux la même certitude que le nôtre. Ces êtres que rejettent la Société à cause de leur désadaptation, vivent dans un monde où l'imagination occupe toute la place, dans un monde de fantaisie et de rêve où tout est permis. Ils réalisent un détournement de réalité et doivent s'expliquer le monde dans leur propre système de référence.

L'exercice de la vie en marge de la Société, les choix de la distanciation et de l'indifférence tactiques de même que le rôle du naïf joué jusqu'à l'absurde font de l'humoriste un persécuté volontaire. Ce paranoïaque consentant se fond au paysage: il devient incongru pour avoir observé les incongruités de la vie, il finit par se croire persécuté à force de jouer le persécuté. Bientôt le guettent la mélancolie, le délire d'imagination et souvent, au bout, la folie.

"L'humeur est la disposition affective fondamentale, riche de toutes les instances émotionnelles instinctives, qui donne à chacun de nos états d'âme une tonalité agréable ou désagréable, oscillant entre deux pôles, l'un pathétique, l'autre apathique (...) L'humeur mélancolique est faite en premier lieu de la douleur morale (...). L'ennui, le spleen, les tristesses qui n'exc/uent pas la délectation morose n'ont qu'un lointain rapport avec la douleur brutale qui terrasse le malade, le faisant vivre nuit et jour dans l'appe/ de la mort (...). Dans la 
mélancolie existe généralement un trouble émotionnel, réalisé par la présence d'une angoisse, (...). Autant la conscience mélancolique est douloureuse, autant la conscience maniaque est euphorique, la joie morbide est icil'antithèse de la douleur morale (...) A cette euphorie pathologique s'associent des modifications du tempo psychique et physique. Le cours des idées est accéléré, les idées s'associent très rapidement, les associations restent sur le plan superficiel, elles se font par assonance, les calembours, les coq-à-l'âne émaillent la conversation du maniaque. Si l'humeur mélancolique est rétractile, le malade apparaissant replié sur sa douleur, l'humeur maniaque est au contraire expansiven (7).

Le poète regarde, il se regarde aussi, il se regarde regardant surtout. II vibre aux comtemplations de sa conscience. II est au centre de sa scène, il ne s'oublie pas, il est rempli d'un orgueil malheureux. Au fond, il force sa lucidité pour découvrir sa nature singulière et se convaincre qu'il est utile (au pire) voire indispensable à d'aucuns (au mieux). Il tourne à l'intérieur de lui-même, il est attentif aux appels de l'instinctif et du vital. Le poète choisit la vulnérabilité et la fréquentation des zones grises de son être. II s'attend à la lumière et défie les douleurs de certaines "visions". II "n'essaye rien... ou va jusqu'au bout», selon la devise d'Alfred Jarry. Nul besoin n'est de démontrer les conséquences de tels voyages tant l'Histoire littéraire abonde en poètes estropiés dans leur tête à force d'engagements dans la nuit...

\section{vers le sacré}

"J'ai monté sur le mur, j'ai appelé le Bon Dieu, II n'a pas répondu. Est-ce que j'étais trop loin?" (Simon, 5 ans) (9)

La poésie est un dialogue de l'homme avec le monde; il y a recherche de la profondeur, de la vibration fondamentale, de la plénitude. II faut franchir le visible pour atteindre à l'invisible derrière le miroir des jours. Toute incursion soutenue dans la Nature, le Temps et l'Espace conduit au sentiment de l'immen- 
sité, de l'inépuisable, du vertige... Ainsi, "le poète écrit de l'autre côté de la vien (Léo Ferré). Parfois, quand on questionne le sensible, c'est le spirituel qui doit répondre.

Et l'enquêteur fréquente clavantage le mystère que la clarté. II fait parler le langage pour qu'il nourisse la flamme de la révélation dans l'obscurité de sa condition. On peut dire du poète comme Mircéa Eliade l'écrit de l'homo religiosus, qu'il "croit toujours qu'il existe une réalité absolue, le sacré, qui transcende ce monde-ci, mais qui s'y manifeste et, de ce fait (...) le rend réel» (11).

Évidemment le sacré n'est pas le religieux. Et le sacré ne serait pas à côté du profane mais au-delà! Curieux, car «le poète croit toujours en un au-delà littéraire" (Léo Ferré). En fait, le poète manifeste le sacré par sa jubilation lorsque quelques lumières lui entrouvent un accès vers I'Absolu. Dans un sens, la poésie permet de prétendre à la pureté, à l'innocence, à l'Étre. Pierre Emmanuel définit même la poésie comme "la langue d'un être à qui il manque l'Étre et qui vise à l'Être en proférant ce manque" (8). Et la multiplicité des fiches signalétiques de cet Être ne change rien à l'Affaire.

"L'homme descend du songe" (Verlaine).

"Mais que foutait Dieu avant la Création?" (Samuel Beckett) (3).

"L'ironie est une culture spécifique de l'esprit et suit l'immédiateté. Puis vient l'homme éthique, puis I'humoriste et finalement l'homme religieux"' (Kirkegaard) (1).

L'humour dont nous parloris n'est pas une figure de rhétorique mais un état d'esprit qui suppose l'impassibilité (flegme ou gravité) et qui ne cherche pas à convaincre de la fausseté de telle ou telle idée, mais à créer un doute sur l'apparence logique du monde. Cette attitude commande le démontage des mécanismes de la réalité objective pour en 
dégager l'insolite; elle transcende le psychologique et le sociologique pour accéder au philosophique et à l'artistique.

Qu'on se le dise, Jésus-Christ lui-même n'a pas dédaigné le calembour, dans une phrase restée célèbre: «Pierre, tu es pierre, et sur cette pierre je bâtirai etc.". L'exemple vient de haut sinon de loin. Freud ne l'avait-il pas pressenti qui énonça que "l'humour a non seulement quelque chose de libérateur (...) mais encore quelque chose de sublime et d'élevé». Et par ailleurs cette définition de l'humour donnée par Ambrose Bierce ne s'appliquerait-elle pas à Dieu, «... un aveugle dans une chambre noire, cherchant un chapeau noir... qui ne s'y trouve pas".

En fait, il se pourrait bien que la démarche du poète repose sur le besoin de retrouver un monde sacré qu'il estime avoir perdu. II veut solenniser ses fulgurances et ses trouvailles, il tient à consacrer lui-même ces moments de grâce en composant des bouquets de langage pour marquer l'événement. La poésie est sacrée comme la prose est profane.

L'humour et le sacré se partagent le domaine de l'esprit. L'un et l'autre utilisent la métaphore des "langues de feun. Comment expliquer que le sourire fleurisse si naturellement au moindre incident dans une église, lieu sacré par excellence? La présence de l'Esprit nourrit le sacré comme la présence d'esprit alimente l'humour. Dieu serait un pur esprit et le Diable un esprit malin (astucieux, roublard?). Pour connaître la Joie éternelle, nous devrions respecter l'esprit et la lettre des textes sacrés. Encore convient-il de savoir lesquels et d'avoir sans cesse l'esprit à ça!

\section{une manière d'existence}

"L'humour poétique, qui est l'art d'éclater de rire en plein pathétique" (Blaise Cendrars).

Pour Camus, l'unique donnée serait l'absurde et le problème serait de savoir comment en sortir. Est absurde ce qui 
est contraire à la logique, au sens commun. L'anxiété est le lot de l'homme prisonnier des contraintes de la civilisation utilitaire et par là, porteuse des nausées de l'absurde.

La démarche humoristique vise la dislocation de la réalité apparente. L'Homme veut exorciser son angoisse en dégonflant les menaces de l'environnement. II doit briser les automatismes confortables de la vie en société. Par l'humour, I'Homme se protège et... attaque.

"La poésie apparaît moins comme une forme d'expression que comme une manière d'exister qui est pour l'Homme la seule existence authentique, la seule qui lui permette d'être intégralement présent dans l'indivision intérieure et la communion avec le monde" (8).

La fête rend plus intensément présentes certaines valeurs. Elle fait éclater le quotidien par une explosion à la fois créatrice et destructrice. La fête exalte la vie. Ainsi, de même, la poésie est une fête de la parole et de l'esprit. Elle désintègre le langage comme s'il s'agissait de la réalité. Il faut des mots acides pour percer l'étoffe de l'existence, des mots-réveils pour mater la grande endormitoire. Sans cesse, le poète oxygène ainsi sa conscience pour allumer ses regards et respirer à hauteur d'homme.

De l'humour et de la poésie. Du langage au sens de la fête, de l'instinct de conservation au désir, du jeu à la folie, du quotidien au sacré. L'humour et la poésie se croisent dans I'Homme et lui donnent une langue et des yeux "additionnels». Ils s'allient pour éclairer son existence de l'intérieur et le rappeler au souvenir de l'Homme primitif.

Et ce n'est pas rien, car après tout, l'imagination (poétique) n'a-t-elle pas été donnée à l'homme pour le consoler de ce qu'il n'est pas, et l'humour, pour le consoler de ce qu'il est... 


\section{RÉFÉRENCES BIBLIOGRAPHIQUES}

(1) ROBERT ESCARPIT, L'humour, PUF, 1967.

(2) MINA ET ANDRÉ GUILLOIS, À l'école du rire, Fayard, 1974.

(3) JEAN PAUL LACROIX, H comme humour, Jacques Grancher, 1983.

(4) HENRI BAUDIN, Boris Vian humoriste, Presses universitaires de Grenoble.

(5) JACQUES BENS, Présence littéraire de Boris Vian, Bordas, 1976.

(6) YVONNE DUPLESSIS, Le surréalisme, PUF, 1969.

(7) J. DELAY ET P. PICHOT, Abrégé de psychologie, Masson et Cie, 1967.

(8) JEAN ONIMUS, La connaissance poétique, Desclée de Brouwer, 1966.

( 9) JEAN-CHARLES, Cancres en liberté, Presses de la Cité, 1968.

(10) NORTHROP FRYE, Pouvoirs de l'imagination, les éditions HMH, 1969.

(11) MIRCÉA ÉLIADE, Le sacré et le profane, Gallimard, 1965. 